\title{
Triple fixed point theorems via $\alpha$-series in partially ordered metric spaces
}

\author{
Ramesh Kumar Vats ${ }^{1}$, Kenan Tas ${ }^{2 *}$, Vizender Sihag ${ }^{1}$ and Amit Kumar ${ }^{1}$
}

\section{*Correspondence:}

kenan@cankaya.edu.tr

${ }^{2}$ Department of Mathematics and

Computer Science, Cankaya

University, Ankara, Turkey

Full list of author information is

available at the end of the article

\begin{abstract}
This manuscript has two aims: first we extend the definitions of compatibility and weakly reciprocally continuity, for a trivariate mapping $F$ and a self-mapping $g$ akin to a compatible mapping as introduced by Choudhary and Kundu (Nonlinear Anal. 73:2524-2531, 2010) for a bivariate mapping $F$ and a self-mapping $g$. Further, using these definitions we establish tripled coincidence and fixed point results by applying the new concept of an $\alpha$-series for sequence of mappings, introduced by Sihag et al. (Quaest. Math. 37:1-6, 2014), in the setting of partially ordered metric spaces.

MSC: $54 \mathrm{H} 25 ; 47 \mathrm{H} 10 ; 54 \mathrm{E} 50$
\end{abstract}

Keywords: $\alpha$-series; compatible mappings; tripled coincidence point; tripled fixed point; partially ordered metric space

\section{Introduction and preliminaries}

The notion of metric space is fundamental in mathematical analysis and the Banach contraction principle is the root of fruitful tree of fixed point theory [1]. In fact, many studies have been done on contractive mappings, e.g., Rhoades [2] presented a comparison of various definitions (more than 100 types varied from 25 basic types) of contractive mappings on complete metric spaces in 1977. See also [3-7]. Up to now, such a study is still going on; proceeding in the same tradition, very recently Sihag et al. [8] introduced the new concept of an $\alpha$-series to give a common fixed point theorem for a sequence of self-mappings. On the other hand, the concept of a coupled fixed point was introduced in 1991 by Chang and Ma [9]. This concept has been of interest to many researchers in metrical fixed point theory (see for example [3,10-15]). Recently, Bhaskar and Lakshmikantham [16] established coupled fixed point theorems for a mixed monotone operator in partially ordered metric spaces. Afterward, Lakshmikantham and Ćirić [17] extended the results of [16] by furnishing coupled coincidence and coupled fixed point theorems for two commuting mappings.

Starting from the background of coupled fixed points, recently Berinde and Borcut [18] introduced the notion of tripled fixed points in partially ordered metric spaces, which refer to the operator as $F: X \times X \times X \rightarrow X$, motivated by the fact that through the coupled fixed point technique we cannot solve a system with the following form:

$$
\left\{\begin{array}{l}
x^{2}+2 y z-6 x+3=0 \\
y^{2}+2 x z-6 y+3=0 \\
z^{2}+2 y x-6 z+3=0 .
\end{array}\right.
$$

(c)2014 Vats et al.; licensee Springer. This is an Open Access article distributed under the terms of the Creative Commons Attribution License (http://creativecommons.org/licenses/by/2.0), which permits unrestricted use, distribution, and reproduction in any medium, provided the original work is properly cited. 
In a subsequent series, Berinde and Borcut [18], introduced the concept of tripled coincidence point and obtained the tripled coincidence point theorems; for more on the tripled fixed point (see [19-27]). Further, Borcut and Berinde [28, 29] established the tripled fixed point theorems by introducing the concept of commuting mappings and also discussed the existence and uniqueness of solution of periodic boundary value problem.

Thus, the purpose of this paper is to prove tripled coincidence and fixed point results in partially ordered metric spaces for a self-mapping $g$ and a sequence $\left\{T_{i}\right\}_{n \in \mathbb{N}}$ of trivariate self-mapping that have some useful properties.

The tripled fixed point theorems we deduce are motivated by the possibilities of solving simultaneous nonlinear equations of the above type.

Now, we collect basic definitions and results regarding coupled and tripled point theory.

Definition 1.1 (see [16]) An element $(x, y) \in X \times X$ is called a coupled fixed point of the mapping $F: X \times X \rightarrow X$ if $F(x, y)=x$ and $F(y, x)=y$.

Definition 1.2 (see [17]) An element $(x, y) \in X \times X$ is called a coupled coincidence point of the mappings $F: X \times X \rightarrow X$ and $g: X \rightarrow X$ if $F(x, y)=g(x)$ and $F(y, x)=g(y)$. In this case, $(g(x), g(y))$ is called a coupled point of coincidence.

Let $(X, \preceq)$ be a partially ordered set and $d$ be a metric on $X$ such that $(X, d)$ is a complete metric space. Consider the product $X \times X \times X$ with the following partial order: for $(x, y, z),(u, v, w) \in X \times X \times X$,

$$
(u, v, w) \preceq(x, y, z) \quad \Leftrightarrow \quad x \succeq u, y \preceq v, z \succeq w .
$$

Definition 1.3 (see [18]) Let $(X, \preceq)$ be a partially ordered set and $F: X \times X \times X \rightarrow X$. We say that $F$ has the mixed monotone property if $F(x, y, z)$ is monotone non-decreasing in $x$ and $z$ and is monotone non-increasing in $y$, that is, for any $x, y, z \in X$

$$
\begin{array}{ll}
x_{1}, x_{2} \in X, \quad x_{1} \preceq x_{2} \quad \Rightarrow \quad F\left(x_{1}, y, z\right) \preceq F\left(x_{2}, y, z\right), \\
y_{1}, y_{2} \in X, \quad y_{1} \preceq y_{2} \quad \Rightarrow \quad F\left(x, y_{1}, z\right) \succeq F\left(x, y_{2}, z\right) \quad \text { and } \\
z_{1}, z_{2} \in X, \quad z_{1} \preceq z_{2} \quad \Rightarrow \quad F\left(x, y, z_{1}\right) \preceq F\left(x, y, z_{2}\right) .
\end{array}
$$

Definition 1.4 (see [18]) We call an element $(x, y, z) \in X \times X \times X$ a tripled fixed point of mapping $F: X \times X \times X \rightarrow X$ if

$$
F(x, y, z)=x, \quad F(y, x, y)=y \quad \text { and } \quad F(z, y, x)=z .
$$

Definition 1.5 (see [18]) Let $(X, d)$ be a complete metric space. It is called metric on $X \times$ $X \times X$, the mapping $d: X \times X \times X \rightarrow X$ with

$$
d[(x, y, z),(u, v, w)]=d(x, u)+d(y, v)+d(z, w) .
$$

Akin to the concept of $g$-mixed monotone property [17] for a bivariate mapping, $F: X \times$ $X \rightarrow X$ and a self-mapping, $g: X \rightarrow X$, Borcut and Berinde [28] introduced the concept of $g$-mixed monotone property for a trivariate mapping $F: X \times X \times X \rightarrow X$ and a selfmapping, $g: X \rightarrow X$ in the following way. 
Definition 1.6 (see [28]) Let $(X, \preceq)$ be a partially ordered set and $F: X \times X \times X \rightarrow X$ and $g: X \rightarrow X$. We say that $F$ has the $g$-mixed monotone property if $F(x, y, z)$ is monotone nondecreasing in $x$ and $z$, and if it is monotone non-increasing in $y$, that is, for any $x, y, z \in X$,

$$
\begin{array}{lll}
x_{1}, x_{2} \in X, & g\left(x_{1}\right) \preceq g\left(x_{2}\right) \quad \Rightarrow \quad F\left(x_{1}, y, z\right) \preceq F\left(x_{2}, y, z\right), \\
y_{1}, y_{2} \in X, & g\left(y_{1}\right) \preceq g\left(y_{2}\right) \quad \Rightarrow \quad F\left(x, y_{1}, z\right) \succeq F\left(x, y_{2}, z\right) \quad \text { and } \\
z_{1}, z_{2} \in X, & g\left(z_{1}\right) \preceq g\left(z_{2}\right) \quad \Rightarrow \quad F\left(x, y, z_{1}\right) \preceq F\left(x, y, z_{2}\right) .
\end{array}
$$

Now, we introduce the concept of compatible mapping for a trivariate mapping $F$ and a self-mapping $g$ akin to compatible mapping as introduced by Choudhary and Kundu [11] for a bivariate mapping $F$ and a self-mapping $g$.

Definition 1.7 Let mapping $F$ and $g$ where $F: X \times X \times X \rightarrow X$ and $g: X \rightarrow X$ are said to be compatible if

$$
\begin{aligned}
& \lim _{n \rightarrow+\infty} d\left(g\left(F\left(x_{n}, y_{n}, z_{n}\right)\right), F\left(g\left(x_{n}\right), g\left(y_{n}\right), g\left(z_{n}\right)\right)\right)=0, \\
& \lim _{n \rightarrow+\infty} d\left(g\left(F\left(y_{n}, x_{n}, y_{n}\right)\right), F\left(g\left(y_{n}\right), g\left(x_{n}\right), g\left(y_{n}\right)\right)\right)=0 \quad \text { and } \\
& \lim _{n \rightarrow+\infty} d\left(g\left(F\left(z_{n}, y_{n}, x_{n}\right)\right), F\left(g\left(z_{n}\right), g\left(y_{n}\right), g\left(x_{n}\right)\right)\right)=0,
\end{aligned}
$$

whenever $\left\{x_{n}\right\},\left\{y_{n}\right\}$, and $\left\{z_{n}\right\}$ are sequences in $X$, such that

$$
\begin{aligned}
& \lim _{n \rightarrow+\infty} F\left(x_{n}, y_{n}, z_{n}\right)=\lim _{n \rightarrow+\infty} g\left(x_{n}\right)=x, \\
& \lim _{n \rightarrow+\infty} F\left(y_{n}, x_{n}, y_{n}\right)=\lim _{n \rightarrow+\infty} g\left(y_{n}\right)=y
\end{aligned}
$$

and

$$
\lim _{n \rightarrow+\infty} F\left(z_{n}, y_{n}, x_{n}\right)=\lim _{n \rightarrow+\infty} g\left(z_{n}\right)=z
$$

for all $x, y, z \in X$.

Definition 1.8 The mappings $F: X \times X \times X \rightarrow X$ and $g: X \rightarrow X$ are called:

(i) Reciprocally continuous if

$$
\begin{aligned}
& \lim _{n \rightarrow+\infty} g\left(F\left(x_{n}, y_{n}, z_{n}\right)\right)=g(x) \text { and } \quad \lim _{n \rightarrow+\infty} F\left(g\left(x_{n}\right), g\left(y_{n}\right), g\left(z_{n}\right)\right)=F(x, y, z), \\
& \lim _{n \rightarrow+\infty} g\left(F\left(y_{n}, x_{n}, y_{n}\right)\right)=g(y) \text { and } \lim _{n \rightarrow+\infty} F\left(g\left(y_{n}\right), g\left(x_{n}\right), g\left(y_{n}\right)\right)=F(y, x, y)
\end{aligned}
$$

and

$$
\lim _{n \rightarrow+\infty} g\left(F\left(z_{n}, y_{n}, x_{n}\right)\right)=g(z) \text { and } \lim _{n \rightarrow+\infty} F\left(g\left(z_{n}\right), g\left(y_{n}\right), g\left(x_{n}\right)\right)=F(z, y, x),
$$

whenever $\left\{x_{n}\right\},\left\{y_{n}\right\}$ and $\left\{z_{n}\right\}$ are sequences in $X$, such that

$$
\begin{aligned}
& \lim _{n \rightarrow+\infty} F\left(x_{n}, y_{n}, z_{n}\right)=\lim _{n \rightarrow+\infty} g\left(x_{n}\right)=x, \\
& \lim _{n \rightarrow+\infty} F\left(y_{n}, x_{n}, y_{n}\right)=\lim _{n \rightarrow+\infty} g\left(y_{n}\right)=y
\end{aligned}
$$


and

$$
\lim _{n \rightarrow+\infty} F\left(z_{n}, y_{n}, x_{n}\right)=\lim _{n \rightarrow+\infty} g\left(z_{n}\right)=z
$$

for some $x, y, z \in X$.

(ii) Weakly reciprocally continuous if

$$
\begin{aligned}
& \lim _{n \rightarrow+\infty} g\left(F\left(x_{n}, y_{n}, z_{n}\right)\right)=g(x) \text { or } \quad \lim _{n \rightarrow+\infty} F\left(g\left(x_{n}\right), g\left(y_{n}\right), g\left(z_{n}\right)\right)=F(x, y, z), \\
& \lim _{n \rightarrow+\infty} g\left(F\left(y_{n}, x_{n}, y_{n}\right)\right)=g(y) \text { or } \lim _{n \rightarrow+\infty} F\left(g\left(y_{n}\right), g\left(x_{n}\right), g\left(y_{n}\right)\right)=F(y, x, y)
\end{aligned}
$$

and

$$
\lim _{n \rightarrow+\infty} g\left(F\left(z_{n}, y_{n}, x_{n}\right)\right)=g(z) \text { or } \lim _{n \rightarrow+\infty} F\left(g\left(z_{n}\right), g\left(y_{n}\right), g\left(x_{n}\right)\right)=F(z, y, x) \text {, }
$$

whenever $\left\{x_{n}\right\},\left\{y_{n}\right\}$ and $\left\{z_{n}\right\}$ are sequences in $X$, such that

$$
\begin{aligned}
& \lim _{n \rightarrow+\infty} F\left(x_{n}, y_{n}, z_{n}\right)=\lim _{n \rightarrow+\infty} g\left(x_{n}\right)=x, \\
& \lim _{n \rightarrow+\infty} F\left(y_{n}, x_{n}, y_{n}\right)=\lim _{n \rightarrow+\infty} g\left(y_{n}\right)=y
\end{aligned}
$$

and

$$
\lim _{n \rightarrow+\infty} F\left(z_{n}, y_{n}, x_{n}\right)=\lim _{n \rightarrow+\infty} g\left(z_{n}\right)=z
$$

for some $x, y, z \in X$.

Definition 1.9 Let $(X, d, \preceq)$ be a partially ordered metric space. We say that $X$ is regular if the following conditions hold:

(i) if a non-decreasing sequence $\left\{x_{n}\right\}$ is such that $x_{n} \rightarrow x$, then $x_{n} \preceq x$ for all $n \geq 0$,

(ii) if a non-increasing sequence $\left\{y_{n}\right\}$ is such that $y_{n} \rightarrow y$, then $y \preceq y_{n}$ for all $n \geq 0$.

Definition 1.10 (see [8]) Let $\left\{a_{n}\right\}$ be a sequence of non-negative real numbers. We say that a series $\sum_{n=1}^{+\infty} a_{n}$ is an $\alpha$-series, if there exist $0<\alpha<1$ and $n_{\alpha} \in \mathbb{N}$ such that $\sum_{i=1}^{k} a_{i} \leq \alpha k$ for each $k \geq n_{\alpha}$.

Remark 1.1 (see [8]) Each convergent series of non-negative real terms is an $\alpha$-series. However, there are also divergent series that are $\alpha$-series. For example, $\sum_{n=1}^{+\infty} \frac{1}{n}$ is an $\alpha$-series.

\section{Main results}

Let $(X, \preceq)$ be a partially ordered set, $g$ be a self-mapping on $X$ and $\left\{T_{i}\right\}_{i \in \mathbb{N}}$ be a sequence of mappings from $X \times X \times X$ into $X$ such that $T_{i}(X \times X \times X) \subseteq g(X)$ and

$$
\begin{aligned}
& T_{i}(x, y, z) \preceq T_{i+1}(u, v, w), \quad T_{i+1}(v, u, v) \preceq T_{i}(y, x, y) \quad \text { and } \\
& T_{i+1}(z, y, x) \preceq T_{i}(w, v, u)
\end{aligned}
$$

for $x, y, z, u, v, w \in X$ with $g(x) \preceq g(u), g(v) \preceq g(y)$ and $g(z) \preceq g(w)$. 
In the proof of our main theorem, we consider sequences that are constructed in the following way.

Let $x_{0}, y_{0}, z_{0} \in X$ be such that $g\left(x_{0}\right) \preceq T_{0}\left(x_{0}, y_{0}, z_{0}\right), g\left(y_{0}\right) \succeq T_{0}\left(y_{0}, x_{0}, y_{0}\right)$ and $g\left(z_{0}\right) \preceq$ $T_{0}\left(z_{0}, y_{0}, x_{0}\right)$. Since $T_{0}(X \times X \times X) \subseteq g(X)$, we can choose $x_{1}, y_{1}, z_{1} \in X$ such that $g\left(x_{1}\right)=T_{0}\left(x_{0}, y_{0}, z_{0}\right), g\left(y_{2}\right)=T_{0}\left(y_{0}, x_{0}, y_{0}\right)$ and $g\left(z_{2}\right)=T_{0}\left(z_{0}, y_{0}, x_{0}\right)$. Again we can choose $x_{2}, y_{2}, z_{2} \in X$ such that $g\left(x_{2}\right)=T_{1}\left(x_{1}, y_{1}, z_{1}\right), g\left(y_{2}\right)=T_{1}\left(y_{1}, x_{1}, y_{1}\right)$ and $g\left(z_{2}\right)=T_{1}\left(z_{1}, y_{1}, x_{1}\right)$. Continuing like this, we can construct three sequences $\left\{x_{n}\right\},\left\{y_{n}\right\}$, and $\left\{x_{n}\right\}$ such that

$$
g\left(x_{n+1}\right)=T_{n}\left(x_{n}, y_{n}, z_{n}\right), \quad g\left(y_{n+1}\right)=T_{n}\left(y_{n}, x_{n}, y_{n}\right) \quad \text { and } \quad g\left(z_{n+1}\right)=T_{n}\left(z_{n}, y_{n}, x_{n}\right)
$$

for all $n \geq 0$.

Now, by using mathematical induction, we prove that

$$
g\left(x_{n}\right) \preceq g\left(x_{n+1}\right), \quad g\left(y_{n}\right) \succeq g\left(y_{n+1}\right) \quad \text { and } \quad g\left(z_{n}\right) \preceq g\left(z_{n+1}\right)
$$

for all $n \geq 0$. Since $g\left(x_{0}\right) \preceq T_{0}\left(x_{0}, y_{0}, z_{0}\right), g\left(y_{0}\right) \succeq T_{0}\left(y_{0}, x_{0}, y_{0}\right)$ and $g\left(z_{0}\right) \preceq T_{0}\left(z_{0}, y_{0}, x_{0}\right)$, in view of $g\left(x_{1}\right)=T_{0}\left(x_{0}, y_{0}, z_{0}\right), g\left(y_{1}\right)=T_{0}\left(y_{0}, x_{0}, z_{0}\right)$ and $g\left(z_{1}\right)=T_{0}\left(z_{0}, y_{0}, x_{0}\right)$, we have $g\left(x_{0}\right) \preceq$ $g\left(x_{1}\right), g\left(y_{0}\right) \succeq g\left(y_{1}\right), g\left(z_{0}\right) \preceq g\left(z_{1}\right)$, that is, (3) holds for $n=0$. We presume that (3) holds for some $n>0$. Now, by (2) and (3), one deduces that

$$
\begin{aligned}
& g\left(x_{n+1}\right)=T_{n}\left(x_{n}, y_{n}, z_{n}\right) \preceq T_{n+1}\left(x_{n+1}, y_{n+1}, z_{n+1}\right)=g\left(x_{n+2}\right), \\
& g\left(y_{n+2}\right)=T_{n+1}\left(y_{n+1}, x_{n+1}, y_{n+1}\right) \preceq T_{n}\left(y_{n}, x_{n}, y_{n}\right)=g\left(y_{n+1}\right)
\end{aligned}
$$

and

$$
g\left(z_{n+1}\right)=T_{n}\left(z_{n}, y_{n}, x_{n}\right) \preceq T_{n+1}\left(z_{n+1}, y_{n+1}, x_{n+1}\right)=g\left(z_{n+2}\right) .
$$

Thus by mathematical induction, we conclude that (3) holds for all $n \geq 0$. Therefore, we have

$$
\begin{aligned}
& g\left(x_{0}\right) \preceq g\left(x_{1}\right) \preceq g\left(x_{2}\right) \preceq \cdots \preceq g\left(x_{n+1}\right) \preceq \cdots, \\
& g\left(y_{0}\right) \succeq g\left(y_{1}\right) \succeq g\left(y_{2}\right) \succeq \cdots \succeq g\left(y_{n+1}\right) \succeq \cdots
\end{aligned}
$$

and

$$
g\left(z_{0}\right) \preceq g\left(z_{1}\right) \preceq g\left(z_{2}\right) \preceq \cdots \preceq g\left(z_{n+1}\right) \preceq \cdots .
$$

In view of the above considerations, we revise Definitions 1.7 and 1.8 as follows.

Definition 2.1 Let $(X, d)$ be a metric space. $\left\{T_{i}\right\}_{i \in \mathbb{N}}$ and $g$ are compatible if

$$
\begin{aligned}
& \lim _{n \rightarrow+\infty} d\left(g\left(T_{n}\left(x_{n}, y_{n}, z_{n}\right)\right), T_{n}\left(g\left(x_{n}\right), g\left(y_{n}\right), g\left(z_{n}\right)\right)\right)=0, \\
& \lim _{n \rightarrow+\infty} d\left(g\left(T_{n}\left(y_{n}, x_{n}, y_{n}\right)\right), T_{n}\left(g\left(y_{n}\right), g\left(x_{n}\right), g\left(y_{n}\right)\right)\right)=0
\end{aligned}
$$


and

$$
\lim _{n \rightarrow+\infty} d\left(g\left(T_{n}\left(z_{n}, y_{n}, x_{n}\right)\right), T_{n}\left(g\left(z_{n}\right), g\left(y_{n}\right), g\left(x_{n}\right)\right)\right)=0,
$$

whenever $\left\{x_{n}\right\},\left\{y_{n}\right\}$ and $\left\{z_{n}\right\}$ are sequences in $X$, such that

$$
\begin{aligned}
& \lim _{n \rightarrow+\infty} T_{n}\left(x_{n}, y_{n}, z_{n}\right)=\lim _{n \rightarrow+\infty} g\left(x_{n+1}\right)=x, \\
& \lim _{n \rightarrow+\infty} T_{n}\left(y_{n}, x_{n}, y_{n}\right)=\lim _{n \rightarrow+\infty} g\left(y_{n+1}\right)=y
\end{aligned}
$$

and

$$
\lim _{n \rightarrow+\infty} T_{n}\left(z_{n}, y_{n}, x_{n}\right)=\lim _{n \rightarrow+\infty} g\left(z_{n+1}\right)=z
$$

for some $x, y, z \in X$.

Definition 2.2 $\left\{T_{i}\right\}_{i \in \mathbb{N}}$ and $g$ are called weakly reciprocally continuous if

$$
\begin{aligned}
& \lim _{n \rightarrow+\infty} g\left(T_{n}\left(x_{n}, y_{n}, z_{n}\right)\right)=g(x), \\
& \lim _{n \rightarrow+\infty} g\left(T_{n}\left(y_{n}, x_{n}, y_{n}\right)\right)=g(y)
\end{aligned}
$$

and

$$
\lim _{n \rightarrow+\infty} g\left(T_{n}\left(z_{n}, y_{n}, x_{n}\right)\right)=g(z)
$$

whenever $\left\{x_{n}\right\},\left\{y_{n}\right\}$, and $\left\{z_{n}\right\}$ are sequences in $X$, such that

$$
\begin{aligned}
& \lim _{n \rightarrow+\infty} T_{n}\left(x_{n}, y_{n}, z_{n}\right)=\lim _{n \rightarrow+\infty} g\left(x_{n+1}\right)=x, \\
& \lim _{n \rightarrow+\infty} T_{n}\left(y_{n}, x_{n}, y_{n}\right)=\lim _{n \rightarrow+\infty} g\left(y_{n+1}\right)=y
\end{aligned}
$$

and

$$
\lim _{n \rightarrow+\infty} T_{n}\left(z_{n}, y_{n}, x_{n}\right)=\lim _{n \rightarrow+\infty} g\left(z_{n+1}\right)=z
$$

for some $x, y, z \in X$.

Now, we establish the main result of this manuscript as follows.

Theorem 2.1 Let $(X, d, \preceq)$ be a partially ordered metric space. Let $g$ be a self-mapping on $X$ and $\left\{T_{i}\right\}_{i \in \mathbb{N}}$ be a sequence of mappings from $X \times X \times X$ into $X$ such that $T_{i}(X \times X \times$ $X) \subseteq g(X), g(X)$ is a complete subset of $X,\left\{T_{i}\right\}_{i \in \mathbb{N}}$ and $g$ are compatible, weakly reciprocally continuous, $g$ is monotonic non-decreasing, continuous, satisfying condition (1) and the following condition:

$$
\begin{aligned}
d\left(T_{i}(x, y, z), T_{j}(u, v, w)\right) \leq & \beta_{i, j}\left[d\left(g(x), T_{i}(x, y, z)\right)+d\left(g(u), T_{j}(u, v, w)\right)\right] \\
& +\gamma_{i, j} d(g(u), g(x))
\end{aligned}
$$


for $x, y, z, u, v, w \in X$ with $g(x) \preceq g(u), g(v) \preceq g(y), g(z) \preceq g(w)$ or $g(x) \succeq g(u), g(v) \succeq g(y)$, $g(z) \succeq g(w) ; 0 \leq \beta_{i, j}, \gamma_{i, j}<1$ for $i, j \in N ; \lim _{n \rightarrow+\infty} \sup \beta_{i, n}<1$. Suppose also that there exists $\left(x_{0}, y_{0}, z_{0}\right) \in X \times X \times X$ such that $g\left(x_{0}\right) \preceq T_{0}\left(x_{0}, y_{0}, z_{0}\right), g\left(y_{0}\right) \succeq T_{0}\left(y_{0}, x_{0}, y_{0}\right)$ and $g\left(z_{0}\right) \preceq$ $T_{0}\left(z_{0}, y_{0}, x_{0}\right)$. If $\sum_{i=1}^{+\infty}\left(\frac{\beta_{i, i+1}+\gamma_{i, i+1}}{1-\beta_{i, i+1}}\right)$ is an $\alpha$-series and $g(X)$ is regular, then $\left\{T_{i}\right\}_{i \in \mathbb{N}}$ and $g$ have a tripled coincidence point, that is, there exists $(x, y, z) \in X \times X \times X$ such that $g(x)=T_{i}(x, y, z)$, $g(y)=T_{i}(y, x, y)$, and $g(z)=T_{i}(z, y, x)$ for $i \in \mathbb{N}$.

Proof We consider the sequences $\left\{x_{n}\right\},\left\{y_{n}\right\}$, and $\left\{z_{n}\right\}$ constructed above and denote $\delta_{n}=$ $d\left(g\left(x_{n}\right), g\left(x_{n+1}\right)\right)+d\left(g\left(y_{n}\right), g\left(y_{n+1}\right)\right)+d\left(g\left(z_{n}\right), g\left(z_{n+1}\right)\right)$. Then, by (4), we get

$$
\begin{aligned}
d\left(g\left(x_{1}\right), g\left(x_{2}\right)\right)= & d\left(T_{0}\left(x_{0}, y_{0}, z_{0}\right), T_{1}\left(x_{1}, y_{1}, z_{1}\right)\right) \\
\leq & \beta_{0,1}\left[d\left(g\left(x_{0}\right), T_{0}\left(x_{0}, y_{0}, z_{0}\right)\right)+d\left(g\left(x_{1}\right), T_{1}\left(x_{1}, y_{1}, z_{1}\right)\right)\right] \\
& +\gamma_{0,1} d\left(g\left(x_{0}\right), g\left(x_{1}\right)\right) \\
= & \beta_{0,1}\left[d\left(g\left(x_{0}\right), g\left(x_{1}\right)\right)+d\left(g\left(x_{1}\right), g\left(x_{2}\right)\right)\right] \\
& +\gamma_{0,1} d\left(g\left(x_{0}\right), g\left(x_{1}\right)\right) .
\end{aligned}
$$

It follows that

$$
\left(1-\beta_{0,1}\right) d\left(g\left(x_{1}\right), g\left(x_{2}\right)\right) \leq\left(\beta_{0,1}+\gamma_{0,1}\right) d\left(g\left(x_{0}\right), g\left(x_{1}\right)\right)
$$

or, equivalently,

$$
d\left(g\left(x_{1}\right), g\left(x_{2}\right)\right) \leq\left(\frac{\beta_{0,1}+\gamma_{0,1}}{1-\beta_{0,1}}\right) d\left(g\left(x_{0}\right), g\left(x_{1}\right)\right) .
$$

Also, one obtains

$$
\begin{aligned}
d\left(g\left(x_{2}\right), g\left(x_{3}\right)\right) & =d\left(T_{1}\left(x_{1}, y_{1}, z_{1}\right), T_{2}\left(x_{2}, y_{2}, z_{2}\right)\right) \\
& \leq\left(\frac{\beta_{1,2}+\gamma_{1,2}}{1-\beta_{1,2}}\right) d\left(g\left(x_{1}\right), g\left(x_{2}\right)\right) \\
& \leq\left(\frac{\beta_{1,2}+\gamma_{1,2}}{1-\beta_{1,2}}\right)\left(\frac{\beta_{0,1}+\gamma_{0,1}}{1-\beta_{0,1}}\right) d\left(g\left(x_{0}\right), g\left(x_{1}\right)\right) .
\end{aligned}
$$

Repeating the above procedure, we have

$$
d\left(g\left(x_{n}\right), g\left(x_{n+1}\right)\right) \leq \prod_{i=0}^{n-1}\left(\frac{\beta_{i, i+1}+\gamma_{i, i+1}}{1-\beta_{i, i+1}}\right) d\left(g\left(x_{0}\right), g\left(x_{1}\right)\right) .
$$

Using similar arguments as above, one can also show that

$$
d\left(g\left(y_{n}\right), g\left(y_{n+1}\right)\right) \leq \prod_{i=0}^{n-1}\left(\frac{\beta_{i, i+1}+\gamma_{i, i+1}}{1-\beta_{i, i+1}}\right) d\left(g\left(y_{0}\right), g\left(y_{1}\right)\right)
$$

and

$$
d\left(g\left(z_{n}\right), g\left(z_{n+1}\right)\right) \leq \prod_{i=0}^{n-1}\left(\frac{\beta_{i, i+1}+\gamma_{i, i+1}}{1-\beta_{i, i+1}}\right) d\left(g\left(z_{0}\right), g\left(z_{1}\right)\right) .
$$


Adding (5), (6), and (7), we have

$$
\begin{aligned}
\delta_{n} & =d\left(g\left(x_{n}\right), g\left(x_{n+1}\right)\right)+d\left(g\left(y_{n}\right), g\left(y_{n+1}\right)\right)+d\left(g\left(z_{n}\right), g\left(z_{n+1}\right)\right) \\
& \leq \prod_{i=0}^{n-1}\left(\frac{\beta_{i, i+1}+\gamma_{i, i+1}}{1-\beta_{i, i+1}}\right)\left[d\left(g\left(x_{0}\right), g\left(x_{1}\right)\right)+d\left(g\left(y_{0}\right), g\left(y_{1}\right)\right)+d\left(g\left(z_{0}\right), g\left(z_{1}\right)\right)\right] \\
& =\prod_{i=0}^{n-1}\left(\frac{\beta_{i, i+1}+\gamma_{i, i+1}}{1-\beta_{i, i+1}}\right) \delta_{0} .
\end{aligned}
$$

Moreover, for $p>0$ and by repeated use of the triangle inequality, one obtains

$$
\begin{aligned}
& d\left(g\left(x_{n}\right), g\left(x_{n+p}\right)\right)+d\left(g\left(y_{n}\right), g\left(y_{n+p}\right)\right)+d\left(g\left(z_{n}\right), g\left(z_{n+p}\right)\right) \\
& \leq d\left(g\left(x_{n}\right), g\left(x_{n+1}\right)\right)+d\left(g\left(y_{n}\right), g\left(y_{n+1}\right)\right)+d\left(g\left(z_{n}\right), g\left(z_{n+1}\right)\right) \\
&+d\left(g\left(x_{n+1}\right), g\left(x_{n+2}\right)\right)+d\left(g\left(y_{n+1}\right), g\left(y_{n+2}\right)\right)+d\left(g\left(z_{n+1}\right), g\left(z_{n+2}\right)\right) \\
&+\cdots+d\left(g\left(x_{n+p-1}\right), g\left(x_{n+p}\right)\right)+d\left(g\left(y_{n+p-1}\right), g\left(y_{n+p}\right)\right)+d\left(g\left(z_{n+p-1}\right), g\left(z_{n+p}\right)\right) \\
& \leq \prod_{i=0}^{n-1}\left(\frac{\beta_{i, i+1}+\gamma_{i, i+1}}{1-\beta_{i, i+1}}\right) \delta_{0}+\prod_{i=0}^{n}\left(\frac{\beta_{i, i+1}+\gamma_{i, i+1}}{1-\beta_{i, i+1}}\right) \delta_{0} \\
&+\cdots+\prod_{i=0}^{n+p-2}\left(\frac{\beta_{i, i+1}+\gamma_{i, i+1}}{1-\beta_{i, i+1}}\right) \delta_{0} \\
&= \sum_{k=0}^{p-1} \prod_{i=0}^{n+k-1}\left(\frac{\beta_{i, i+1}+\gamma_{i, i+1}}{1-\beta_{i, i+1}}\right) \delta_{0}=\sum_{k=n}^{n+p-1} \prod_{i=0}^{k-1}\left(\frac{\beta_{i, i+1}+\gamma_{i, i+1}}{1-\beta_{i, i+1}}\right) \delta_{0} .
\end{aligned}
$$

Let $\alpha$ and $n_{\alpha}$ be as in Definition 1.10, then, for $n \geq n_{\alpha}$, and using the fact that the geometric mean of non-negative numbers is less than or equal to the arithmetic mean, it follows that

$$
\begin{aligned}
& d\left(g\left(x_{n}\right), g\left(x_{n+p}\right)\right)+d\left(g\left(y_{n}\right), g\left(y_{n+p}\right)\right)+d\left(g\left(z_{n}\right), g\left(z_{n+p}\right)\right) \\
& \leq \sum_{k=n}^{n+p-1}\left[\frac{1}{k} \sum_{i=0}^{k-1}\left(\frac{\beta_{i, i+1}+\gamma_{i, i+1}}{1-\beta_{i, i+1}}\right)\right]^{k} \delta_{0} \\
& \leq\left(\sum_{k=n}^{n+p-1} \alpha^{k}\right) \delta_{0} \\
& \leq \frac{\alpha^{n}}{1-\alpha} \delta_{0} .
\end{aligned}
$$

Now, taking the limit as $n \rightarrow+\infty$, one deduces that

$$
\lim _{n \rightarrow+\infty}\left[d\left(g\left(x_{n}\right), g\left(x_{n+p}\right)\right)+d\left(g\left(y_{n}\right), g\left(y_{n+p}\right)\right)+d\left(g\left(z_{n}\right), g\left(z_{n+p}\right)\right)\right]=0,
$$

which further implies that

$$
\lim _{n \rightarrow+\infty} d\left(g\left(x_{n}\right), g\left(x_{n+p}\right)\right)=\lim _{n \rightarrow+\infty} d\left(g\left(y_{n}\right), g\left(y_{n+p}\right)\right)=\lim _{n \rightarrow+\infty} d\left(g\left(z_{n}\right), g\left(z_{n+p}\right)\right)=0
$$


Thus $\left\{g\left(x_{n}\right)\right\},\left\{g\left(y_{n}\right)\right\}$ and $\left\{g\left(z_{n}\right)\right\}$ are Cauchy sequences in $X$. Since $g(X)$ is complete, then there exists $(r, s, t) \in X \times X \times X$, with $g(r)=x, g(s)=y$ and $g(t)=z$, such that

$$
\begin{aligned}
& \lim _{n \rightarrow+\infty} g\left(x_{n+1}\right)=\lim _{n \rightarrow+\infty} T_{n}\left(x_{n}, y_{n}, z_{n}\right)=x, \\
& \lim _{n \rightarrow+\infty} g\left(y_{n+1}\right)=\lim _{n \rightarrow+\infty} T_{n}\left(y_{n}, x_{n}, y_{n}\right)=y
\end{aligned}
$$

and

$$
\lim _{n \rightarrow+\infty} g\left(z_{n+1}\right)=\lim _{n \rightarrow+\infty} T_{n}\left(z_{n}, y_{n}, x_{n}\right)=z
$$

Now, as $\left\{T_{i}\right\}_{i \in \mathbb{N}}$ and $g$ are weakly reciprocally continuous, we have

$$
\lim _{n \rightarrow+\infty} g\left(T_{n}\left(x_{n}, y_{n}, z_{n}\right)\right)=g(x), \quad \lim _{n \rightarrow+\infty} g\left(T_{n}\left(y_{n}, x_{n}, y_{n}\right)\right)=g(y)
$$

and

$$
\lim _{n \rightarrow+\infty} g\left(T_{n}\left(z_{n}, y_{n}, x_{n}\right)\right)=g(z)
$$

On the other hand, the compatibility of $\left\{T_{i}\right\}_{i \in \mathbb{N}}$ and $g$ yields

$$
\begin{aligned}
& \lim _{n \rightarrow+\infty} d\left(g\left(T_{n}\left(x_{n}, y_{n}, z_{n}\right)\right), T_{n}\left(g\left(x_{n}\right), g\left(y_{n}\right), g\left(z_{n}\right)\right)\right)=0, \\
& \lim _{n \rightarrow+\infty} d\left(g\left(T_{n}\left(y_{n}, x_{n}, y_{n}\right)\right), T_{n}\left(g\left(y_{n}\right), g\left(x_{n}\right), g\left(y_{n}\right)\right)\right)=0
\end{aligned}
$$

and

$$
\lim _{n \rightarrow+\infty} d\left(g\left(T_{n}\left(z_{n}, y_{n}, x_{n}\right)\right), T_{n}\left(g\left(z_{n}\right), g\left(y_{n}\right), g\left(x_{n}\right)\right)\right)=0 \text {. }
$$

Then we have

$$
\begin{aligned}
& \lim _{n \rightarrow+\infty} T_{n}\left(g\left(x_{n}\right), g\left(y_{n}\right), g\left(z_{n}\right)\right)=g(x), \\
& \lim _{n \rightarrow+\infty} T_{n}\left(g\left(y_{n}\right), g\left(x_{n}\right), g\left(y_{n}\right)\right)=g(y)
\end{aligned}
$$

and

$$
\lim _{n \rightarrow+\infty} T_{n}\left(g\left(z_{n}\right), g\left(y_{n}\right), g\left(x_{n}\right)\right)=g(z)
$$

Since $\left\{g\left(x_{n}\right)\right\}$ and $\left\{g\left(z_{n}\right)\right\}$ are non-decreasing and $\left\{g\left(y_{n}\right)\right\}$ is non-increasing, using the regularity of $X$, we have $g\left(x_{n}\right) \preceq x, y \preceq g\left(y_{n}\right)$ and $g\left(z_{n}\right) \preceq z$ for all $n \geq 0$. Then by (4), one obtains

$$
\begin{aligned}
d\left(T_{i}(x, y, z), T_{n}\left(g\left(x_{n}\right), g\left(y_{n}\right), g\left(z_{n}\right)\right)\right) \leq & \beta_{i, n}\left[d\left(g(x), T_{i}(x, y, z)\right)\right. \\
& \left.+d\left(g\left(g\left(x_{n}\right)\right), T_{n}\left(g\left(x_{n}\right), g\left(y_{n}\right), g\left(z_{n}\right)\right)\right)\right] \\
& +\gamma_{i, n} d\left(g\left(g\left(x_{n}\right)\right), g(x)\right) .
\end{aligned}
$$


Taking the limit as $n \rightarrow+\infty$, we obtain $T_{i}(x, y, z)=g(x)$ as $\beta_{i, n}<1$. Similarly, it can be proved that $g(y)=T_{i}(y, x, y)$ and $g(z)=T_{i}(z, y, x)$. Thus, $(x, y, z)$ is a tripled coincidence point of $\left\{T_{i}\right\}_{i \in \mathbb{N}}$ and $g$.

Now, we give useful conditions for the existence and uniqueness of a tripled common fixed point.

Theorem 2.2 In addition to the hypotheses of Theorem 2.1, suppose that the set of coincidence points is comparable with respect to $g$, then $\left\{T_{i}\right\}_{i \in \mathbb{N}}$ and $g$ have a unique tripled common fixed point, that is, there exists $(x, y, z) \in X \times X \times X$ such that $x=g(x)=T_{i}(x, y, z)$, $y=g(y)=T_{i}(y, x, y)$, and $z=g(z)=T_{i}(z, y, x)$ for $i \in \mathbb{N}$.

Proof From Theorem 2.1, the set of tripled coincidence points is non-empty. Now, we show that if $(x, y, z)$ and $(r, s, t)$ are tripled coincidence points, that is, if $g(x)=T_{i}(x, y, z)$, $g(y)=T_{i}(y, x, y), g(z)=T_{i}(z, y, x), g(r)=T_{i}(r, s, t), g(s)=T_{i}(s, r, s)$, and $g(t)=T_{i}(t, s, r)$, then $g(x)=g(r), g(y)=g(s)$ and $g(z)=g(t)$. Since the set of coincidence points is comparable, applying condition (4) to these points, we get

$$
\begin{aligned}
d(g(x), g(r)) & =d\left(T_{i}(x, y, z), T_{j}(r, s, t)\right) \\
& \leq \beta_{i, j}\left[d\left(g(x), T_{i}(x, y, z)\right)+d\left(g(r), T_{j}(r, s, t)\right)\right]+\gamma_{i, j} d(g(r), g(x)),
\end{aligned}
$$

and so as $\gamma_{i, j}<1$, it follows that $d(g(x), g(r))=0$, that is, $g(x)=g(r)$. Similarly, it can be proved that $g(y)=g(s)$ and $g(z)=g(t)$. Hence, $\left\{T_{i}\right\}_{i \in \mathbb{N}}$ and $g$ have a unique tripled point of coincidence. It is well known that two compatible mappings are also weakly compatible, that is, they commute at their coincidence points. Thus, it is clear that $\left\{T_{i}\right\}_{i \in \mathbb{N}}$ and $g$ have a unique tripled common fixed point whenever $\left\{T_{i}\right\}_{i \in \mathbb{N}}$ and $g$ are weakly compatible. This finishes the proof.

If $g$ is the identity mapping, as a consequence of Theorem 2.1, we state the following corollary.

Corollary 2.3 Let $(X, d, \preceq)$ be a complete partially ordered metric space. Let $\left\{T_{i}\right\}_{i \in \mathbb{N}}$ be a sequence of mappings from $X \times X \times X$ into $X$ such that $\left\{T_{i}\right\}_{i \in \mathbb{N}}$ satisfies, for $x, y, z, u, v, w \in X$, with $x \preceq u, v \preceq y, z \preceq w$ or $u \preceq x, y \preceq v$, and $w \preceq z$, the following conditions:

(i) $T_{n}(x, y, z) \preceq T_{n+1}(u, v, w)$,

(ii) $d\left(T_{i}(x, y, z), T_{j}(u, v, w)\right) \leq \beta_{i, j}\left[d\left(x, T_{i}(x, y, z)\right)+d\left(u, T_{j}(u, v, w)\right)\right]+\gamma_{i, j} d(u, x)$, with $0 \leq \beta_{i, j}, \gamma_{i, j}<1$ and $i, j \in \mathbb{N}$.

Suppose also that there exists $\left(x_{0}, y_{0}, z_{0}\right) \in X \times X \times X$ such that $x_{0} \preceq T_{0}\left(x_{0}, y_{0}, z_{0}\right), y_{0} \succeq$ $T_{0}\left(y_{0}, x_{0}, y_{0}\right)$ and $z_{0} \preceq T_{0}\left(z_{0}, y_{0}, z_{0}\right)$. If $\sum_{i=1}^{+\infty}\left(\frac{\beta_{i, i+1}+\gamma_{i, i+1}}{1-\beta_{i, i+1}}\right)$ is an $\alpha$-series and $X$ is regular, then $\left\{T_{i}\right\}_{i \in \mathbb{N}}$ has a tripled fixed point, that is, there exists $(x, y, z) \in X \times X \times X$ such that $x=$ $T_{i}(x, y, z), y=T_{i}(y, x, y)$ and $z=T_{i}(z, y, x)$, for $i \in N$.

Example 2.3 Take $X=[0,1]$ endowed with usual metric $d=|x-y|$ for all $x, y \in X$ and $\preceq$ be defined as 'greater than/equal to' the $(X, d, \preceq)$ be partial order metric space. Let $T_{i}: X^{3} \rightarrow$ $X$ be mapping defined as $T_{i}(x, y, z)=\frac{x+y+z}{3 i} ; i \in \mathbb{N}$ and $g$ is self-mapping defined as $g(x)=x$.

Clearly, $T_{i}(x, y, z) \subseteq g(X), g(X)$ is a complete subset of $X$. 
By choosing the sequences $\left\{x_{n}\right\}=\frac{1}{n},\left\{y_{n}\right\}=\frac{1}{n+1}$ and $\left\{z_{n}\right\}=\frac{1}{n+2}$, one can easily observe that $\left\{T_{i}\right\}_{i \in \mathbb{N}}$ and $g$ are compatible, weakly reciprocally continuous; $g$ is monotonic nondecreasing, continuous, as well as satisfying condition (1).

Again by taking $0<\beta_{i, j}<1$ and $0 \leq \gamma_{i, j}<1$, it is easy to check inequality (4) holds, thus all the hypotheses of Theorem 2.1 are satisfied and $(0,0,0),(1,1,1)$ are the tripled coincident points of $g$ and $T_{i}$. Moreover, using the same $T_{i}$ and $g$ in Theorem 2.2, $(0,0,0)$ is the unique fixed point of $g$ and $T_{i}$.

Remark 2.1 Open problem: In this paper, we prove tripled fixed point results. The idea can be extended to multidimensional cases. But the technicalities in the proofs therein will be different. We consider this as an open problem.

\section{Competing interests}

The authors declare that they have no competing interests.

Authors' contributions

All authors contributed equally to this work. All authors read and approved the final manuscript.

\section{Author details}

${ }^{1}$ Department of Mathematics, National Institute of Technology, Hamirpur, 177005, India. ${ }^{2}$ Department of Mathematics and Computer Science, Cankaya University, Ankara, Turkey.

\section{Acknowledgements}

The authors gratefully acknowledge the learned referees for providing a suggestion to improve the manuscript. The first author also acknowledges the Council of Scientific and Industrial Research, Government of India, for providing financial assistance under research project no. 25(0197)/11/EMR-II.

Received: 29 November 2013 Accepted: 23 April 2014 Published: 12 May 2014

\section{References}

1. Agarwal, RP, Meehan, M, O'Regan, D: Fixed Point Theory and Application. Cambridge University Press, Cambridge (2001)

2. Rhoades, BE: A comparison of various definitions of contractive mappings. Trans. Am. Math. Soc. 226(2), 257-290 (1977)

3. Luong, NV, Thuan, NX: Coupled fixed point theorems in partially ordered metric spaces. Bull. Math. Anal. Appl. 2, 16-24 (2010)

4. Nieto, JJ, Rodriguez-Lopez, R: Contractive mapping theorems in partially ordered sets and applications to ordinary differential equation. Order 22, 223-239 (2005)

5. Nieto, JJ, Rodriguez-Lopez, R: Existence and uniqueness of fixed point in partially ordered sets and applications to ordinary differential equations. Acta Math. Sin. Engl. Ser. 23(12), 2205-2212 (2007)

6. Paesano, D, Vetro, P: Suzuki's type characterizations of completeness for partial metric spaces and fixed points for partially ordered metric spaces. Topol. Appl. 159(3), 911-920 (2012)

7. Ran, ACM, Reurings, MCB: A fixed point theorem in partially ordered sets and some applications to matrix equations. Proc. Am. Math. Soc. 132, 1435-1443 (2004)

8. Sihag, V, Vetro, C, Vats, RK: A fixed point theorem in G-metric spaces via $\alpha$-series. Quaest. Math. 37, 1-6 (2014)

9. Chang, SS, Ma, YH: Coupled fixed point for mixed monotone condensing operators and an existence theorem of the solutions for a class of functional equations arising in dynamic programming. J. Math. Anal. Appl. 160, 468-479 (1991)

10. Agarwal, RP, Kadelburg, Z, Radenovic, S: On coupled fixed point results in asymmetric G-metric spaces. J. Inequal. Appl. 2013, $528(2013)$

11. Choudhary, BS, Kundu, A: A coupled coincidence point result in partially ordered metric spaces for compatible mappings. Nonlinear Anal. 73, 2524-2531 (2010)

12. Berinde, $\mathrm{V}$ : Generalized coupled fixed point theorems for mixed monotone mappings in partially ordered metric spaces. Nonlinear Anal. 74, 7347-7355 (2010)

13. Samet, B: Coupled fixed point theorems for a generalized Meir-Keeler contraction in partially ordered metric spaces. Nonlinear Anal. 72, 4508-4517 (2010)

14. Karapinar, E, Agarwal, RP: Further fixed point results on G-metric spaces. Fixed Point Theory Appl. 2013,154 (2013)

15. Agarwal, RP, Karapinar, E: Remarks on some coupled fixed point theorems in G-metric spaces. Fixed Point Theory Appl. 2013, 2 (2013)

16. Bhaskar, TG, Lakshmikantham, V: Fixed point theorems in partially ordered metric space and applications. Nonlinear Anal. 65, 1379-1393 (2006)

17. Lakshmikantham, V, Cirić, L: Coupled fixed point theorems for nonlinear contractions in partially ordered metric spaces. Nonlinear Anal. 70, 4341-4349 (2009)

18. Berinde, V, Borcut, M: Tripled fixed point theorems for contractive type mappings in partially ordered metric spaces. Nonlinear Anal. 74(15), 4889-4897 (2011) 
19. Aydi, H, Karapinar, E, Shatanawi, W: Tripled common fixed point results for generalized contractions in ordered generalized metric spaces. Fixed Point Theory Appl. 2012, 101 (2012)

20. Aydi, H, Karapinar, E, Vetroc, C: Meir-Keeler type contractions for tripled fixed points. Acta Math. Sci. 32(6), 2119-2130 (2012)

21. Aydi, H, Karapinar, E, Shatanawi, W: Tripled fixed point results in generalized metric spaces. J. Appl. Math. 2012, Article ID 314279 (2012)

22. Aydi, H, Karapinar, E, Postolache, M: Tripled coincidence point theorems for weak $\varphi$-contractions in partially ordered metric spaces. Fixed Point Theory Appl. 2012, 44 (2012)

23. Aydi, H, Karapinar, E, Radenovic, S: Tripled coincidence fixed point results for Boyd-Wong and Matkowski type contractions. Rev. R. Acad. Cienc. Exactas Fís. Nat., Ser. a Mat. 107(2), 339-353 (2013)

24. Aydi, H, Abbas, M, Sintunavarat, W, Kumam, P: Tripled fixed point of $W$-compatible mappings in abstract metric spaces. Fixed Point Theory Appl. 2012, 134 (2012)

25. Abbas, M, Ali, B, Sintunavarat, W, Kumam, P: Tripled fixed point and tripled coincidence point theorems in intuitionistic fuzzy normed spaces. Fixed Point Theory Appl. 2012, 187 (2012)

26. Abbas, M, Aydi, H, Karapinar, E: Tripled fixed points of multi-valued nonlinear contraction mappings in partially ordered metric spaces. Abstr. Appl. Anal. 2011, Article ID 812690 (2011)

27. Karapinar, E, Aydi, H, Mustafa, Z: Some tripled coincidence point theorems for almost generalized contractions in ordered metric spaces. Tamkang J. Math. 44(3), 233-251 (2013)

28. Borcut, $M$, Berinde, $V$ : Tripled coincidence theorems for contractive type mappings in partially ordered metric spaces. Appl. Math. Comput. 218(10), 5929-5936 (2012)

29. Borcut, M: Tripled coincidence theorems for contractive type mappings in partially ordered metric spaces. Appl. Math. Comput. 218(14), 7339-7346 (2012)

10.1186/1029-242X-2014-176

Cite this article as: Vats et al.: Triple fixed point theorems via $\alpha$-series in partially ordered metric spaces. Journal of Inequalities and Applications 2014, 2014:176

\section{Submit your manuscript to a SpringerOpen ${ }^{\ominus}$ journal and benefit from:}

- Convenient online submission

Rigorous peer review

- Immediate publication on acceptance

- Open access: articles freely available online

- High visibility within the field

- Retaining the copyright to your article 